\title{
Virtual Inertia Control of D-PMSG Based on the Principle of Active Disturbance Rejection Control
}

\author{
Qiaoming Shi ${ }^{\dagger}$, Gang Wang*, Lijun Fu*, Yang Liu*, You Wu* and Li Xu*
}

\begin{abstract}
The virtual inertia control (VIC) of wind turbine with directly-driven permanent-magnet synchronous generator (D-PMSG) can act similarly to the conventional synchronous generator in inertia response and frequency control, thereby supporting the system frequency stability. However, because the wind speed is inconstant and changeable to a certain extent and the D-PMSG is a complex nonlinear system, there are great difficulties in the virtual inertia optimal control of the D-PMSG. Based on the design principle of the active disturbance rejection control (ADRC), this paper presents a new VIC strategy for the D-PMSG from the perspective of power disturbance suppression in the system. The strategy helps fulfill the power grid disturbance estimation and compensation by means of the extended state observer (ESO) so as to improve the disturbance-resisting performance of the system. Compared with conventional proportional-derivative virtual inertia control (PDVIC), this method, which is of better adaptability and robustness, can not only improve the property of the DPMSG responding to the system frequency but also reduce the influence of wind speed disturbance. The simulation and experiment results have verified the effectiveness and feasibility of the VIC based on the ADRC.
\end{abstract}

Keywords: Wind turbine generator, Virtual inertia control, Active disturbance rejection control, Frequency response, Robustness

\section{Nomenclature}

$\omega_{n} \quad$ Rated angular frequency of the power system $(\mathrm{rad} / \mathrm{s})$.

$\omega$ Angular frequency of the power system ( $\mathrm{rad} / \mathrm{s})$.

$\Delta \omega$ Angular frequency deviation of the system $(\mathrm{rad} / \mathrm{s})$.

$H \quad$ Inertia time constant of the power system (s).

$D \quad$ Damping coefficient of the load.

$S_{B} \quad$ Total capacity of the synchronous generators (Watt).

$P_{m} \quad$ Output power of prime motor (Watt).

$P_{L} \quad$ Frequency-insensitive load of system (Watt).

$R p \quad$ Frequency droop coefficient of the system.

$J_{w} \quad$ Rotational inertia of the D-PMSG $\left(\mathrm{kg} \cdot \mathrm{m}^{2}\right)$.

$\omega_{w} \quad$ Angular velocity of the wind turbine $(\mathrm{rad} / \mathrm{s})$.

$P_{w} \quad$ Grid-connected power of the D-PMSG (Watt).

$P_{M P P T}$ Output power of MPPT control (Watt).

$P_{V I C} \quad$ Auxiliary output power of VIC (Watt).

$k_{p}, k_{d}$ Control parameters of PDVIC.

$R \quad$ Blade radius $(\mathrm{m})$.

$V \quad$ Wind speed $(\mathrm{m} / \mathrm{s})$.

$C_{p} \quad$ Power coefficient.

$\theta \quad$ Blade pitch angle (degree).

$\lambda \quad$ Tip speed ratio.

$x, x_{1}, x_{2}$ State variable.

$\dagger$ Corresponding Author: College of Electrical Engineering, Xi'an Jiaotong University, China. (shqm14210@163.com)

* National Key Laboratory of Vessel Integrated Power System Technology, Naval University of Engineering, China. (wanggang60742@ 126.com, lijunfu2006@sina.cn, \{liuyang8930,wuyou1351\}@126.com, xuli199108@163.com)

Received: December 16, 2014; Accepted: June 18, 2015 $z_{1}, z_{2}$ Observed quantity of the state variable.

$\beta_{1}, \beta_{2}$ Control parameters of ESO.

$h \quad$ Control step of the ADRC.

$k_{0}, k_{V I C}$ Control parameters of ADRVIC.

Subscripts and Superscripts

ref Reference value.

$0 \quad$ Initial value.

$n \quad$ Rated value.

* $\quad$ Per unit value.

\section{Introduction}

The wind turbine with D-PMSG, which has low failure rate, high reliability and high power generation efficiency, has gradually become a dominant model in wind power industry [1]. At present, it can perform the maximum power point tracking (MPPT) control through the fullpower converter (FPC) for an efficient use of wind power. However, the rotating speed of the permanent-magnet synchronous generator (PMSG) is decoupled from the system frequency in this control mode, while the output power and rotating speed of D-PMSG only vary with wind speed without responding to the frequency fluctuation. Thus, the system cannot be provided inertia and frequency support. With a continuous increase in wind power penetration, the number of connected conventional power plants will reduce. The decoupling effect of D-PMSG will lead to a continuous decrease of system inertia only to have 
a serious influence on the frequency stability of the system [2-4].

To enable wind turbine generators to give power support to the system like traditional synchronous generators when the frequency fluctuates, a deload control method for wind turbine generators was proposed in references [5-7]. In spite of improving the ability of the wind turbine generators to be involved in the frequency control, this method is not economical when large-scale wind power connected to the grid, with the inertia time constant of MW-level wind turbine generators up to $6 \mathrm{~s}$ [2] and the kinetic energy stored in the rotor being considerable in normal operating conditions of the turbine. References [2, 8] and [9] offered a frequency response control method based on the kinetic energy of the rotor in wind turbines. The method introduces the auxiliary power related to the proportional and derivative terms of the frequency deviation on the basis of the MPPT control. When the system frequency drops, the D-PMSG can release the kinetic energy stored in the rotor to get into the system inertia response and primary frequency control. Because the converter can adjust the active power quickly, the wind turbine generators can provide greater virtual inertia than their inherent inertia [2]. Therefore, this control method is also called PDVIC. Using the PDVIC, it is possible for the D-PMSG to adjust the power output and participate in the frequency regulation when the frequency fluctuates and to quit the frequency response when the system is stable. Both enabling the D-PMSG to be involved in the frequency response of the system and ensuring the electricitygenerating efficiency, this method has become the focal point of research.

The frequency response ability of the D-PMSG is easily affected by the wind speed and the control parameters of the unit in practical operation. To bring the frequency response ability of units into full play under different working conditions, reference [10] has analyzed the frequency response ability of the D-PMSG and proposed a method of optimizing and improving the frequency control of wind turbine generators based on PDVIC. The method can improve the frequency support ability of wind turbine generators to some extent. References [11] and [12] have proposed a smooth control method of wind farms power output based on the inertia control. The method can increase the frequency support time of the wind farm through the coordinated control of multiple units. However, the influence of the wind speed random disturbance has not been taken into account in [11]. Reference [13] has offered a design method of primary frequency control of wind turbine generators based on the fuzzy control principle. Although confirmed through experiments, the method still needs to be used for power reserve. Reference [13] only confirmed the characteristics of the wind turbine generators responding to the frequency deviation of the system but it did not confirm the relation between the output of the units and the frequency deviation under VIC.
The ADRC is a new control method, which overcomes the shortcomings of classic PID control [14]. The method can transform the nonlinear system into the linear integrator series structure through the dynamic compensation linearization so as to simplify the control design and eliminate the dependence upon the model of the controlled system. And it can implement the system disturbance estimation and compensation by use of the ESO so as to improve the ability to restrain the error and disturbance. Thus, it is of better adaptability and robustness $[15,16]$.

In this paper, the active disturbance rejection virtual inertia control (ADRVIC) of the D-PMSG is proposed from the perspective of power disturbance rejection of the system according to the principle of ADRC. Firstly, a simplified model of the wind power integrated system (WPIS) is established for the design of ADRVIC. Then, the process of design and the method of applying the ADRVIC are presented based on this model. Finally, the effectiveness and feasibility of the ADRVIC are verified by comparing the ADRVIC and the PDVIC through the simulation and experiment system.

\section{Principle of Conventional Virtual Inertia Control}

The imbalance of generating power and load will lead to the change of frequency in power systems, while the mechanical inertia of the rotor in the traditional synchronous generator has a critical influence on the speed of variation in the system frequency. The principle is expressed as [17]:

$$
\frac{d \Delta \omega^{*}}{d t}=\frac{\Delta P_{A}^{*}-D \Delta \omega^{*}}{2 H}
$$

where $H$ is the inertia time constant of the system (s); $D$ is the damping coefficient of the load; $\Delta \omega^{*}$ is the angular frequency deviation of the system (pu), $\Delta \omega^{*}=\omega^{*}-\omega_{\mathrm{n}}{ }^{*}$, where $\omega^{*}$ is the angular frequency of the system $(\mathrm{pu}), \omega_{\mathrm{n}}{ }^{*}$ is the rated angular frequency of the system $(\mathrm{pu}), \omega_{\mathrm{n}}{ }^{*}=1$; $\Delta P_{A}{ }^{*}$ is the unbalance power of the system (pu), $\Delta P_{A}{ }^{*}=P_{m}{ }^{*}+P_{w}{ }^{*}-P_{L}{ }^{*}$, where $P_{m}{ }^{*}$ and $P_{L}{ }^{*}$ are the output power of prime motor and the frequency-insensitive load, respectively (pu); $P_{w}{ }^{*}=P_{w} / S_{B}$, where $P_{w}$ is the gridconnected power of the D-PMSG (Watt), $S_{B}$ is the total capacity of the synchronous generators $(\mathrm{V} \cdot \mathrm{A})$. Before the VIC is introduced into the D-PMSG, the D-PMSG operates in the MPPT control mode. At this time, $P_{w}{ }^{*}=P_{M P P T}{ }^{*}$, where $P_{M P P T}{ }^{*}=P_{M P P T} / S_{B}, P_{M P P T}$ is the output power of D-PMSG in the MPPT control mode (Watt).

As shown in (1), the greater $H$ is, the more slowly the system frequency changes, so the more stable the system is under the same unbalance power.

Because of the decoupling effect of the FPC, the DPMSG in the power system in a normal MPPT control 
mode cannot release or absorb the rotor kinetic energy like traditional synchronous generators when the system frequency fluctuates. Its rotational kinetic energy is "hidden" and almost has no contribution to the inertia of the system.

The conventional PDVIC makes the D-PMSG change its output when the system frequency fluctuates mainly through the introduction of the auxiliary power related to the proportional and derivative terms of the system frequency deviation so as to simulate the properties of primary frequency control and inertia response of the traditional synchronous generators. The principle is expressed as [10]:

$$
P_{V I C}=-k_{p} \Delta \omega^{*}-k_{d} \frac{d \Delta \omega^{*}}{d t}
$$

where $P_{V I C}$ is the auxiliary output power of VIC (Watt); $k_{p}$ and $k_{d}$ are the proportional and derivative parameters, $k_{p}>0$, $k_{d}>0$ in practical projects.

After the VIC is introduced, the grid-connected power of the D-PMSG is equal to the sum of the output power of MPPT control and VIC if the response time of the DPMSG is ignored, that is

$$
P_{w}=P_{M P P T}+P_{V I C}
$$

So the response equation of the system frequency turns from (1) into:

$$
\frac{d \Delta \omega^{*}}{d t}=\frac{\left(P_{m}^{*}+P_{M P P T}^{*}-P_{L}^{*}\right)+P_{V I C}^{*}-D \Delta \omega^{*}}{2 H}
$$

where $P_{V I C}{ }^{*}=P_{V I C} / S_{B}$.

Combining with (2), (4) can be derived as follows:

$$
\frac{d \Delta \omega^{*}}{d t}=\frac{\left(P_{m}^{*}+P_{M P P T}^{*}-P_{L}^{*}\right)-\left(k_{p}^{*}+D\right) \Delta \omega^{*}}{2\left(H+k_{d}^{*}\right)}
$$

where $k_{p}^{*}=k_{p} / S_{B}, k_{d}^{*}=k_{d} / S_{B}$.
As is shown in (5), the conventional PDVIC improves the stability of the system from the aspects of inertia and damping.

The PDVIC is simple in principle and clear in physical meaning, but it has difficulty in adjusting the parameters $k_{p}$ and $k_{d}$ and has poor adaptability under wind speed disturbance. Therefore, based on the design of ADRC, this paper has proposed a new VIC strategy from the perspective of reducing the disturbance of power.

\section{Active Disturbance Rejection Virtual Inertia Control Method}

\subsection{Mathematical model of the wind power integrated system}

The system structure of the D-PMSG which has been connected to the grid is shown in Fig. 1. The D-PMSG contains the wind turbine, PMSG [18], FPC and its control systems.

Wind energy is captured through the wind wheel to drive the PMSG. Its rotor movement equation is expressed as:

$$
J_{w} \frac{d \omega_{w}}{d t}=\frac{P_{w i n d}}{\omega_{w}}-\frac{P_{w}}{\omega_{w}}
$$

where $J_{w}$ is the rotational inertia of the D-PMSG $\left(\mathrm{kg} \cdot \mathrm{m}^{2}\right)$, $\omega_{w}$ is the angular velocity of the wind turbine $(\mathrm{rad} / \mathrm{s}), P_{\text {wind }}$ is the mechanical power which the wind turbine obtains from the wind (Watt). $P_{\text {wind }}$ is expressed as [19]:

$$
\begin{gathered}
P_{\text {wind }}=0.5 \rho \pi R^{2} v^{3} C_{p}(\lambda, \theta) \\
\lambda=\omega_{w} R / v
\end{gathered}
$$

where $\rho$ is the air density $\left(\mathrm{kg} / \mathrm{m}^{3}\right), R$ is blade radius $(\mathrm{m}), v$ is wind speed $(\mathrm{m} / \mathrm{s}), C_{p}$ is the power coefficient, $\theta$ is blade pitch angle (degree), and $\lambda$ is tip speed ratio.

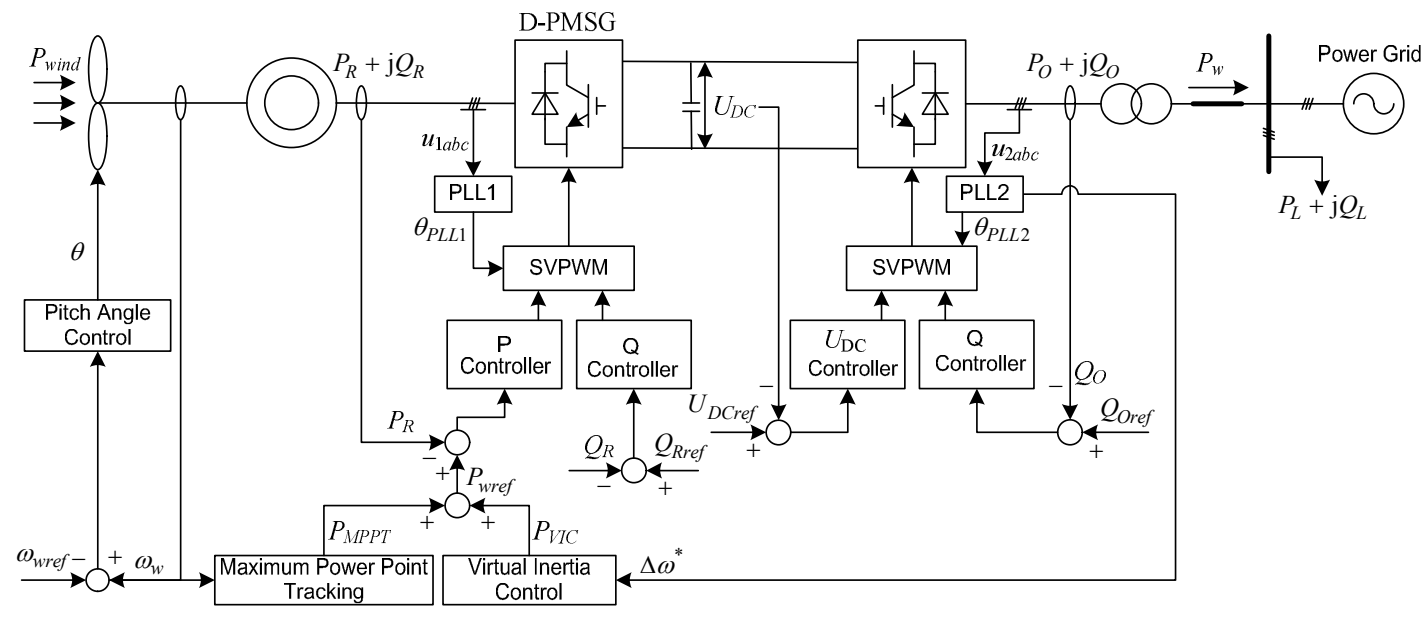

Fig. 1. WPIS with D-PMSG 
Normally, when the output power of the unit is less than the limited power, the blade pitch angle will be adjusted to zero. At this time, $C_{p}$ is only a function of $\lambda$. $C_{p}$ can reach its maximum $C_{\text {pmax }}$ by adjusting $\omega_{w}$ thus to fulfill the MPPT control. At this time the corresponding $\lambda$ equals the optimal top speed ratio $\lambda_{\text {opt }}$. The power extracted from the wind is expressed as $[19,20]$ :

$$
P_{M P P T}=k_{\max } \omega_{w}^{3}
$$

where $k_{\max }$ is the coefficient used for the wind turbine to extract the maximum energy, $k_{\max }=0.5 \rho \pi R^{5} C_{\mathrm{pmax}} / \lambda_{\mathrm{opt}}{ }^{3}$.

When the power system is in a steady state, the DPMSG operates in MPPT mode, with the auxiliary power $P_{V I C}$ equals zero. When the system frequency fluctuates, the virtual inertia controller starts working. It adjusts the rotating speed and power output of the generator and regulates the rotor kinetic energy through the fast power control of the FPC, so that the D-PMSG will be involved in the power system frequency regulation to damp the frequency fluctuation.

In the process of VIC, whether the D-PMSG can participate in VIC is also related to its rotating speed. When the rotating speed of D-PMSG is low, the kinetic energy of the rotor is relatively low, and releasing rotor kinetic energy may lead to unit halting. When the rotating speed is relatively high, keeping increasing rotor kinetic energy may make the unit's rotating speed exceed the speed limit. In order to ensure the stable operation of the D-PMSGs, when the rotating speed of a unit is lower than $0.6 \mathrm{pu}$ or exceeds $1.1 \mathrm{pu}, P_{V I C}$ is adjusted to zero and the unit will temporarily quit from VIC. Therefore, under different operating conditions, the reference power of VIC is set as follows [4]:

$$
P_{V I C}= \begin{cases}0, & \omega_{w}<0.6 \omega_{w n} \\ f\left(\Delta \omega^{*}\right), & 0.6 \omega_{w n}<\omega_{w}<1.1 \omega_{w n} \\ 0, & 1.1 \omega_{w n}<\omega_{w}\end{cases}
$$

where $\omega_{w n}$ is the rated angular velocity of the wind turbine $(\mathrm{rad} / \mathrm{s})$.

When the larger wind speed causes the rotating speed to be greater than its rated value, the pitch angle control starts acting to increase the pitch angle and decrease the mechanical power obtained from the wind, thereby reducing the rotating speed. In this process, the output power of the unit will reach the upper limit of the FPC, which is generally determined as $1.0 \sim 1.2 \mathrm{pu}$. The pitch angle controller is usually a PI controller.

\subsection{Simplified model of the wind power integrated system}

The wind power integrated system is a complex nonlinear system. Discussing the VIC method based on its detailed model will complicate the design of the controller. In ADRC, the unmodeled dynamics of the system can be regarded as internal disturbance and be compensated by the ESO. Therefore, a simplified model of the wind power integrated system can be built based on the physical principles of VIC. And based on the simplified model the design of ARDVIC can be discussed.

Both the D-PMSG and the traditional synchronous generator will participate in the adjustment of the system frequency after the VIC is introduced. The structure of the system shown in Fig. 1 can be simplified as shown in Fig. 2.

The VIC is mainly related to the electromechanical transient process of the system, so the electromagnetic transient process in the D-PMSG system can be ignored in the simplified model. In references [9] and [21], the DPMSG and FPC are equivalent to an inertia unit and their electromagnetic loss power is ignored, then

$$
P_{w}=\frac{1}{T_{A} s+1} P_{w r e f}
$$

where $s$ is the Laplace operator; $P_{\text {wref }}$ is the reference power of the D-PMSG (Watt), $P_{\text {wref }}=P_{M P P T}+P_{V I C} ; T_{A}$ is the inertia time constant of the PMSG and the FPC (s), its value being about $0.2 \mathrm{~s}$. As the process of the system frequency response is commonly $10 \sim 30 \mathrm{~s}$, the dynamics can be ignored in the study of system frequency response control. The output power of the D-PMSG is supposed to follow the variation in reference power strictly, that is,

$$
P_{w}=P_{w r e f}
$$

The above analysis shows that in the study of the DPMSG VIC, the response time of the D-PMSG can be ignored in Eq. (3).

Fig. 2 shows that there is a certain relation between the active power component and the frequency of the system, which is as follows:

$$
\Delta P_{m}^{*}+\Delta P_{w}^{*}-\Delta P_{L}^{*}=\Delta \omega^{*}(2 H s+D)
$$

where $\Delta P_{m}{ }^{*}=P_{m}{ }^{*}-P_{m 0}{ }^{*}, \Delta P_{w}{ }^{*}=P_{w}{ }^{*}-P_{w 0}{ }^{*}, \Delta P_{L}{ }^{*}=P_{L}{ }^{*}-P_{L 0}{ }^{*}$, $P_{m 0}{ }^{*}, P_{w 0}{ }^{*}$ and $P_{L 0}{ }^{*}$ are the original power of prime motor,

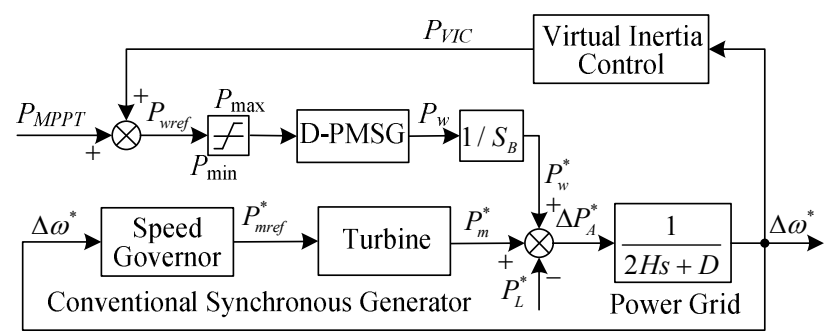

Fig. 2. Simplified structure of WPIS with VIC 
D-PMSG and frequency-insensitive load respectively when the system is in a steady state, the relation of them is expressed as:

$$
P_{m 0}^{*}+P_{w 0}^{*}-P_{L 0}^{*}=0
$$

As for the D-PMSG, the amount of power change is expressed as:

$$
\Delta P_{w}^{*}=\Delta P_{M P P T}^{*}+\Delta P_{V I C}^{*}
$$

where $\Delta P_{V I C}{ }^{*}=P_{V I C}{ }^{*}-P_{V I C O}{ }^{*}, \Delta P_{M P P T}=P_{M P P T}{ }^{*}-P_{M P P T O}{ }^{*}, P_{V I C O}{ }^{*}$ and $P_{\text {MPPTO }}{ }^{*}$ are the VIC output power and the MPPT output power respectively when the system is in a steady state. At this time, the virtual inertia controller does not operate; that is, $P_{V I C O}{ }^{*}=0$, so

$$
\Delta P_{V I C}^{*}=P_{V I C}^{*}
$$

Supposing $\Delta P^{*}=\Delta P_{m}{ }^{*}+\Delta P_{M P P T}{ }^{*}-\Delta P_{L}{ }^{*}-D \Delta \omega^{*}$, the dynamic response equation of the system frequency which includes the VIC can be shown below based on (13), (15) and (16):

$$
\frac{d \Delta \omega^{*}}{d t}=\frac{\Delta P^{*}}{2 H}+\frac{P_{V I C}^{*}}{2 H}
$$

By $x=\Delta \omega^{*}$, then (17) is derived as follows:

$$
\left\{\begin{array}{l}
\dot{x}=g(x, w(t))+b u \\
y=x
\end{array}\right.
$$

where $w(t)=\Delta P^{*} / 2 H, g(x, w(t))=w(t), u(t)=P_{V I C}{ }^{*}, b=1 / 2 H$, $w(t)$ is the external disturbance, $g(x, w(t))$ represents the combined effect of internal dynamics and external disturbance [14, 15], $x$ is the state variable, $u$ is the control variable, $y$ is the output variable, $b$ is the system parameter.

As shown in (18), the frequency response of the WPIS containing D-PMSG can be simplified as a first-order system, and the fluctuations of wind speed and load in this system can be treated as the disturbance of the system.

\subsection{Active disturbance rejection virtual inertia controller}

The design of the active disturbance rejection virtual inertia controller is mainly based on the nonlinear system shown in (18). The nonlinear system control design is complicated while the linear system control design is relatively simple. The core of the ADRC is to implement the real-time observation of system disturbance with the ESO and compensate the disturbance in the control variable so as to realize the linearization. Supposing the accurate estimation of the total disturbance of the system $g(x, w(t))$ can be made, its value is expressed as:

$$
z_{2} \approx g(x, w(t))
$$

Then the disturbance observation can be introduced into the control system as the compensated control variable:

$$
u=u_{0}-z_{2} / b
$$

where $u_{0}$ is the control variable without disturbance compensation.

By the action of control variable $u$, the nonlinear system shown in (18) turns into:

$$
\left\{\begin{array}{l}
\dot{x} \approx b u_{0} \\
y=x
\end{array}\right.
$$

Now the nonlinear system in (18) is transformed into a linear system as shown in (21), the process of which is called dynamic compensation linearization. For the observation of the system disturbance, the state variable is firstly extended in the system shown in (18) to obtain the following expression:

$$
\left\{\begin{array}{l}
\dot{x}_{1}=x_{2}+b u \\
\dot{x}_{2}=G(t) \\
y=x_{1}
\end{array}\right.
$$

where $x_{1}=x, x_{2}$ is the extended state variable, $x_{2}=g(x, w(t))$, $G(t)$ is the unknown function. The real-time observation of $x_{1}$ and $x_{2}$ depends on the ESO. Reference [14] offered the method of designing the ESO. The algorithm is as follows:

$$
\left\{\begin{array}{l}
e=z_{1}-y \\
f e=f a l(e, 0.5, \delta), \quad f e_{1}=f a l(e, 0.25, \delta) \\
\dot{z}_{1}=z_{2}+b_{0} u-\beta_{1} f e \\
\dot{z}_{2}=-\beta_{2} f e_{1}
\end{array}\right.
$$

where $e$ is the tracking error of ESO, $z_{1}$ and $z_{2}$ are the observed quantity of the state variable $x_{1}$ and $x_{2}$ respectively, $\beta_{1}$ and $\beta_{2}$ are the control parameters, and $b_{0}$ is the factor of the disturbance compensation. When $b$ is known, $b_{0=} b$; when $b$ is unknown, $b_{0}$ takes the estimated value of $b . f e, f e_{1}$ and $f a l(e, \alpha, \delta)$ are nonlinear function of $e$ [14]:

$$
f a l(e, \alpha, \delta)= \begin{cases}\frac{e}{\delta^{1-\alpha}}, & |e| \leq \delta \\ |e|^{\alpha} \operatorname{sign}(e), & |e|>\delta>0\end{cases}
$$

where $\alpha$ and $\delta$ are constants.

If $\alpha=1, f a l(e, \alpha, \delta)$ will equal $e$. At this time, $e$ can be used instead of $f e$ and $f e_{1}$ in (23), thus to simplify (23) and obtain the following expression [14]: 


$$
\left\{\begin{array}{l}
e=z_{1}-y \\
\dot{z}_{1}=z_{2}+b_{0} u-\beta_{1} e \\
\dot{z}_{2}=-\beta_{2} e
\end{array}\right.
$$

Here is the linear implementation method of the ESO. After the state variable is extended, (21) will be turned into:

$$
\left\{\begin{array}{l}
\dot{x}_{1} \approx b_{0} u_{0} \\
y=x_{1}
\end{array}\right.
$$

The ADRC aims at allowing $x_{1}$ tend to the reference value $\Delta \omega_{r}{ }^{*}$, where $\Delta \omega_{r}{ }^{*}=0$. As a result, the control variable $u_{0}$ in (26) is

$$
u_{0}=k_{0}\left(\Delta \omega_{r}^{*}-x_{1}\right)
$$

where $k_{0}$ is the control parameter. For $z_{1}$ is the observed quantity of $x_{1}$, so it can replace $x_{1}$. The result is expressed as:

$$
u_{0}=k_{0}\left(\Delta \omega_{r}^{*}-z_{1}\right)
$$

which is called linear state error feedback control law (LSEFL) of the system.

The final control variable can be derived from (20):

$$
u=u_{0}-z_{2} / b_{0}
$$

Based on (25) and (28-29), it is possible to obtain the expression for the first-order linear active disturbance rejection virtual inertia controller of the WPIS as following:

$$
\left\{\begin{array}{l}
e=z_{1}-y \\
\dot{z}_{1}=z_{2}+b_{0} u-\beta_{1} e \\
\dot{z}_{2}=-\beta_{2} e \\
\varepsilon=\Delta \omega_{r}^{*}-z_{1} \\
u_{0}=k_{0} \varepsilon \\
u=u_{0}-z_{2} / b_{0}
\end{array}\right.
$$

The structure of the controller in (30) is shown in Fig. 3. It shows that wind turbine generators can participate in the close-loop control of system frequency through

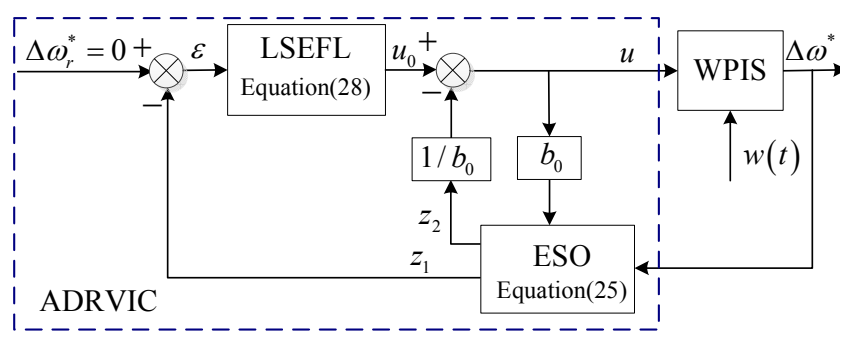

Fig. 3. Structure of ADRVIC
ADRVIC. As for the stability of the close-loop system, based on reference [22] it can be proved that if $g(x, w(t))$ is bounded, which means there is a positive number $M_{1}$ and $|g(x, w(t))| \leq M_{1}$, there will be $\beta_{1}>0, \beta_{2}>0$ and $k_{0}>0$ making the close-loop BIBO stable. The detail proof process of the conclusion is shown in references [15] and [22].

\subsection{Application of the ADRVIC}

The adopted algorithm needs to be discretized in practical application. The discretization method of (30) can be expressed as [23]:

$$
\left\{\begin{array}{l}
e(k)=z_{1}(k)-y(k) \\
z_{1}(k+1)=z_{1}(k)+h\left(z_{2}(k)+b_{0} u(k)-\beta_{1} e(k)\right) \\
z_{2}(k+1)=z_{2}(k)+h\left(-\beta_{2} e(k)\right) \\
\varepsilon(k)=\Delta \omega_{r}^{*}-z_{1}(k) \\
u_{0}(k)=k_{0} \varepsilon(k) \\
u(k)=u_{0}(k)-z_{2}(k) / b_{0}
\end{array}\right.
$$

where $h$ is the control step of the controller. The smaller the value $h$ takes, the stronger the control ability of the system is. $h$ usually takes the value between 0.0001 and 0.1 for the linear ESO [23].

The control parameters $\beta_{1}$ and $\beta_{2}$ can be adjusted according to the bandwidth of ESO $\omega_{o}$ [24]. Deriving the characteristic equation of ESO from Eq. (25) and set its poles as $\omega_{o}$, as in the following equation:

$$
\lambda(s)=s^{2}+\beta_{1} s+\beta_{2}=\left(s+\omega_{o}\right)^{2}
$$

From Eq. (32) the following equation can be obtained:

$$
\left\{\begin{array}{l}
\beta_{1}=2 \omega_{o} \\
\beta_{2}=\omega_{o}^{2}
\end{array}\right.
$$

Large $\omega_{o}$ can improve the performance of ESO, but it will also magnify the system noise. In practice, a compromise is made between the speed at which the observer tracks the states and its sensitivity to the sensor noises [24].

For the first-order system, $\omega_{o}$ is set as $1 / 2 h$, and Eq. (33) turns into [23]:

$$
\left\{\begin{array}{l}
\beta_{1}=1 / h \\
\beta_{2}=1 /\left(4 h^{2}\right)
\end{array}\right.
$$

The control parameter $k_{0}$ can be set according to the bandwidth of the feedback system [24]:

$$
k_{0}=\omega_{c}
$$

where $\omega_{c}$ is the system bandwidth, $\omega_{c}=1 / 5 \sim 1 / 3 \omega_{o}$. 
However, in ADRVIC, parameters $u_{0}$ and $k_{0}$ both have actual physical meanings. If the tracking error of ESO is ignored, then $z_{1} \approx \Delta \omega^{*}$. Considering $\Delta \omega_{r}{ }^{*}=0$, Eq. (28) turns into:

$$
u_{0} \approx-k_{0} \Delta \omega^{*}
$$

In power systems, the relation between the variation of conventional synchronous generator's power output and the variation of system frequency is as follows [17]:

$$
\Delta P_{G}^{*}=-\Delta \omega^{*} / R_{p}
$$

where $\Delta P_{G}{ }^{*}$ is the power output variation of synchronous generator (pu), and $R_{p}$ is the frequency droop coefficient of the system.

In Eq. (30), the meaning of $u_{0}$ is the same as $\Delta P_{G}{ }^{*}$, it represents the variation of generator's power output when system frequency changes, and $k_{0}$ represents the proportional relation between the variation of generator's power output and the frequency deviation. Thus in analogy to Eq. (37) the following equation is obtained:

$$
k_{0}=1 / R_{p}
$$

In power systems, $R_{p}=0.02 \sim 0.06$ [17], thus $k_{0}=16.7 \sim$ 50.0 in (38).

The control variable obtained from (31) practically refers to all the generators in the system, including all the D-PMSG and traditional synchronous generators, while $P_{V I C}$ is concerned with the single D-PMSG, so the interrelationship of the D-PMSG capacity and the system capacity needs to be considered. Suppose the system has $n$ synchronous generators and $m$ D-PMSGs, each D-PMSG with a power rating of $P_{D-P M S G}$. For the $j$ th D-PMSG:

$$
\begin{gathered}
P_{V I C, j}=k_{V I C, j} u \\
k_{V I C, j}=P_{w n, j} S_{B} /\left(\sum_{i=1}^{n} S_{S G, i}+\sum_{j=1}^{m} P_{w n, j}\right)
\end{gathered}
$$

where $S_{S G, i}$ is the rated capacity of the $i$ th traditional synchronous generator, $P_{w n, j}$ is the rated capacity of the $j$ th D-PMSG, $k_{V I C, j}$ is the allocation coefficient of the $j$ th DPMSG. According to the definition, $S_{B}=\sum S_{S G, i}$.

Eq. (40) shows that by using the allociation coefficient, the capacities of both D-PMSGs and synchronous generators are considered, thus the coordinated control of D-PMSGs and conventional synchronous generators is implemented.

According to (30) and (39), the power of the D-PMSG participating in the system frequency response is expressed as:

$$
P_{V I C, j}=-k_{V I C, j} k_{0} z_{1}-k_{V I C, j} z_{2} / b_{0}
$$

In (41), $z_{1}$ and $z_{2}$ are the observed quantities of $\Delta \omega^{*}$ and $g(x, w(t))$ respectively, $g(x, w(t))$ contains the derivative term of the system frequency $d \Delta \omega^{*} / d t$, so (41) is expressed as:

$$
\begin{gathered}
P_{V I C, j}=-k_{V I C, j} k_{0} \Delta \omega^{*}-k_{V I C, j} g(x, w(t)) / b_{0} \\
\approx-k_{A D R C 1, j} \Delta \omega^{*}-k_{A D R C 2, j} \frac{d \Delta \omega^{*}}{d t}
\end{gathered}
$$

where $k_{A D R C 1, j}=k_{V I C, j} k_{0}, k_{A D R C 2, j}=k_{V I C, j} / b_{0}$.

The comparison of (42) and (2) indicates that the added auxiliary power in D-PMSG is composed of the proportional and derivative terms of the system frequency deviation. Therefore the ADRVIC has the same basic function as the PDVIC so that the D-PMSG can achieve the properties of frequency control similar to the traditional synchronous generators. However, what is worthy of note is that this algorithm incorporates the disturbances of the wind speed, D-PMSG and grid into the total disturbance for the purpose of observation and compensation and that it is more suitable for wind turbine generators due to its strong ability of disturbance resisting.

\section{Simulation Study}

\subsection{Simulation platform}

To verify the effectiveness of ADRVIC, a test system built on Matlab/Simulink is used for simulation study. As shown in Fig. 4, the system includes an equivalent wind farm and an equivalent thermal power plant. The wind farm with an installed capacity of $8 \mathrm{MW}$ has four $2 \mathrm{MW}$ rated D-PMSGs while the thermal power plant with an installed capacity of 8.20 MW has one synchronous generator. In the system, the power of load0 and that of load1 are $10.20+\mathrm{j} 3.1 \mathrm{MVA}$ and $1.12+\mathrm{j} 0.20$ MVA respectively. Load0 is fixed and load1 is variable. S1 is used for the switching of load1. With the penetration of wind power is up to $49.3 \%$, the system is a weak AC system.

In the simulation system, the main parameters of the wind turbine generator are: rated power $P_{w n}=2 \mathrm{MW}$, rated rotating speed $\omega_{w n}=1.65 \mathrm{rad} / \mathrm{s}$, radius of the wind wheel $R=42 \mathrm{~m}$, rated wind speed $v_{n}=11 \mathrm{~m} / \mathrm{s}$, and inertia time constant $H_{\mathrm{w}}=4.5 \mathrm{~s}$. The capacity of the synchronous generator is $S_{n}=8.2 \mathrm{MVA}$, and the inertia time constant is

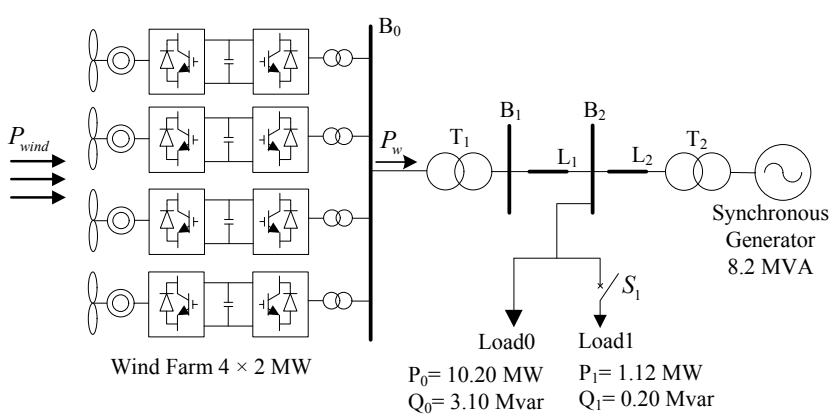

Fig. 4. Single-line diagram of simulation system 
$H=6 \mathrm{~s}$. The parameters of PDVIC are: $k_{p}=20 \times 10^{6}, k_{d}=$ $30 \times 10^{6}[2,9]$. The parameters of ADRVIC are: $h=0.01$, $\beta_{1}=100, \beta_{2}=2500, k_{0}=40, b_{0}=1 / 12$.

\subsection{Case 1: sudden load changes}

This case refers to the inertia response of the system when there is a sudden change in load under the condition of constant wind speed which is $10.7 \mathrm{~m} / \mathrm{s}$. In the simulation system, as the D-PMSG units are of the same type, the response characteristic of each unit is also the same. Fig. 5 and Fig. 6 show the dynamic response of the system at the time when the variable load is switched in or out at $5 \mathrm{~s}$. In the figures, $\omega, P_{w}, \omega_{w}$ and $\theta$ denote the angular frequency of the system, the output of the D-PMSG, the angular velocity and the blade pitch angle of the wind turbine respectively. The response curves marked in black, red and blue respectively represent three different situations, in which the ADRVIC and PDVIC are introduced and the
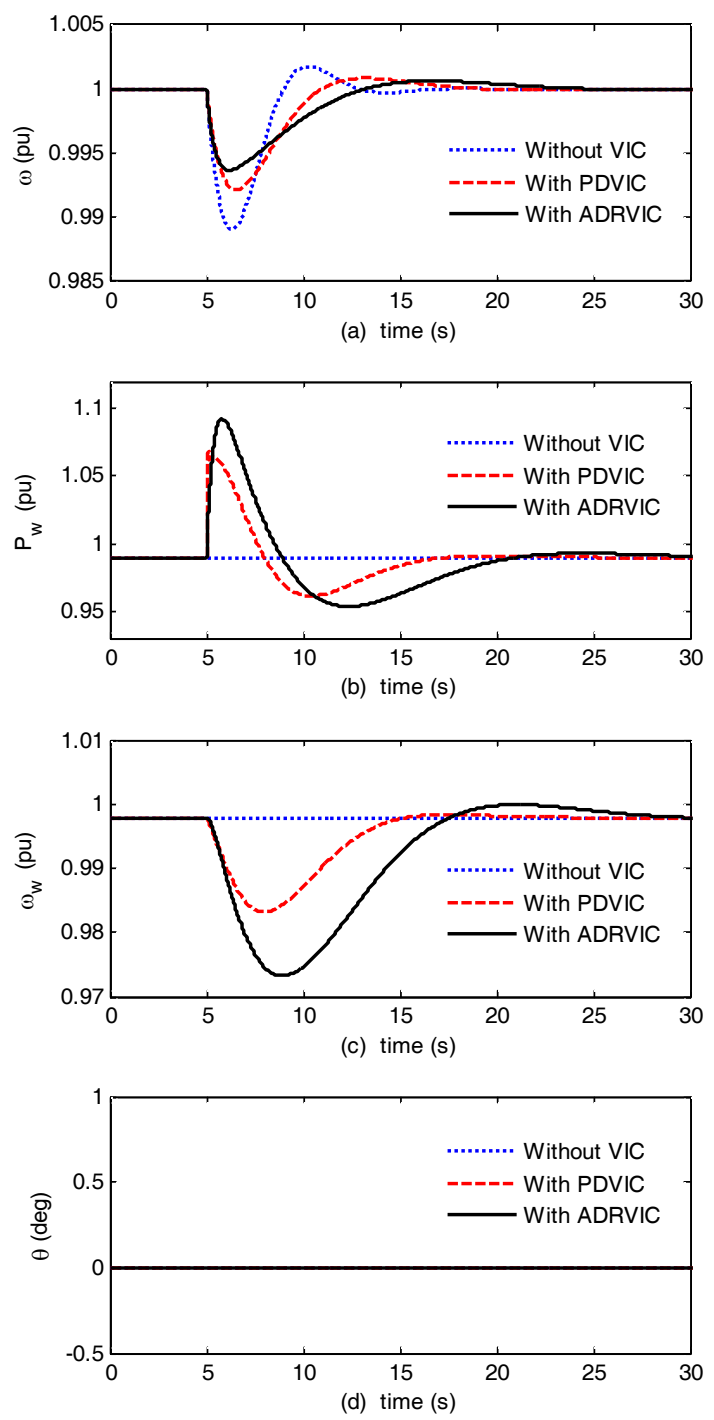

Fig. 5. System inertia response for a sudden increase in load
VIC is not.

As shown in Fig. 5, the variable load is switched in suddenly. Fig. 5(a) shows that the decreasing speed and magnitude of the system frequency are improved significantly with ADRVIC and PDVIC. Compared with PDVIC, ADRVIC improves more. Fig. 5(b) and Fig. 5(c) show that D-PMSG releases the kinetic energy of the rotor to support the system frequency by increasing the output of the unit when frequency drops. Compared with PDVIC, ADRVIC can take full advantage of the inertia of the system and release the frequency regulation potential of the unit so as to improve the property of the system frequency.

As shown in Fig. 6, the variable load is switched out suddenly. Fig. 6(a) shows that ADRVIC can effectively reduce the rise rate and magnitude of the system frequency and is better than PDVIC in control performance. Fig. 6(b) and Fig. 6(c) show that D-PMSG increases the kinetic energy of the rotor to support the system frequency by decreasing the output of the unit when frequency rises. Fig.
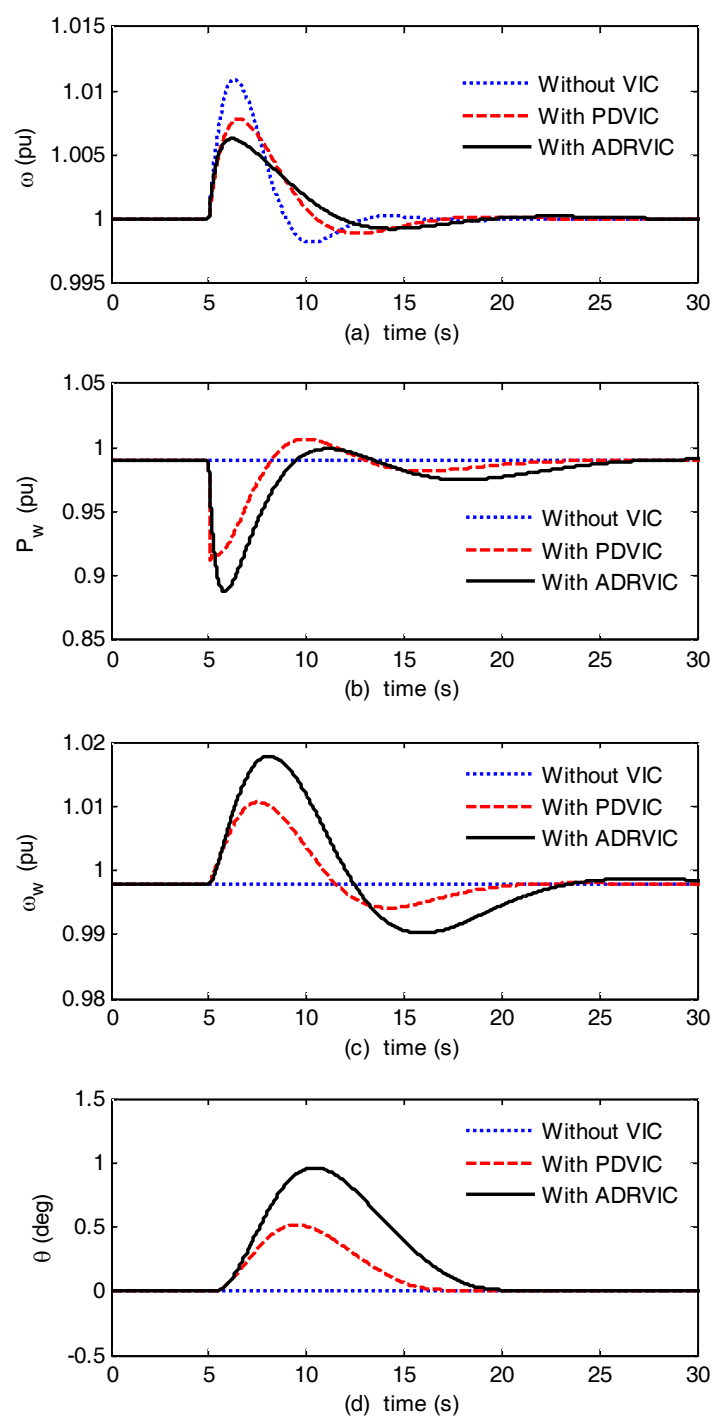

Fig. 6. System inertia response for a sudden decrease in load 

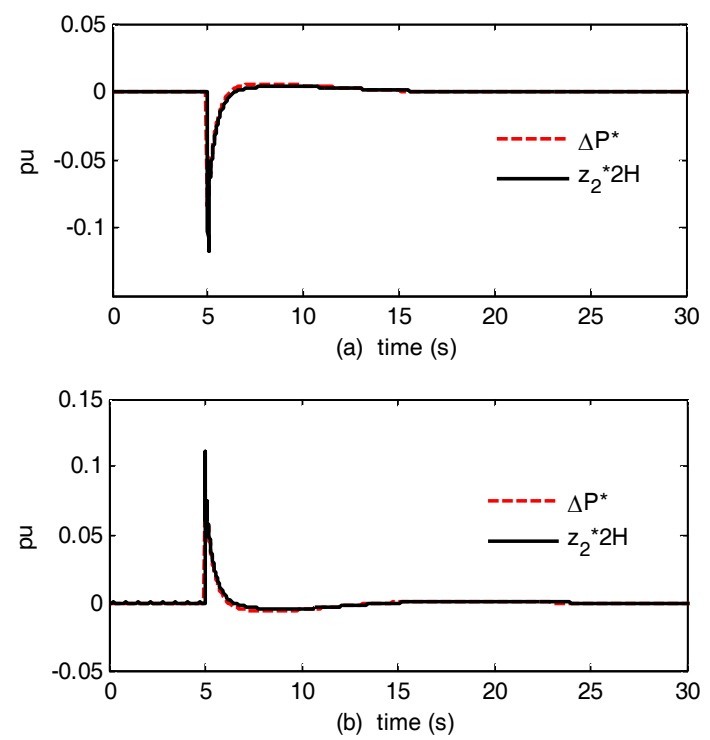

Fig. 7. Observed effect of ESO in ADRVIC at the time of a sudden rise or drop in load

6(d) shows that the pitch angle control starts to increase the pitch angle and decrease the power extracted from the wind so as to stop the rotating speed of the unit from further rising when it exceeds the rated speed during the period of the D-PMSG participating in the system frequency response.

As shown in Fig. 5 and Fig. 6, the adjustment of the output and rotating speed of units through VIC can make them participate in the system frequency response and support the stability of system frequency when load disturbance causes the frequency to fluctuate. When the system is stable, the VIC will quit operation and the units will return to the MPPT control mode to put wind energy into full use. It is obvious that the introduction of VIC helps establish a new coupling relationship between the rotor and the system frequency, but the relationship does not affect the efficiency of power generation.

Fig. 7(a) and Fig. 7(b) show the contrast effect of observation of ESO in ADRVIC respectively at the time of a sudden rise or drop in load. In the figures, the red curve denotes the practical power disturbance while black curve denotes the observed effect of ESO: $\Delta P^{*}=g(x, w(t)) \times 2 H, z_{2}$ is the observation of $g(x, w(t))$. Fig. 7 shows that the ESO can track and estimate the system disturbance accurately under load disturbance, which provides a reliable basis for the compensation and suppression of power system disturbance.

\subsection{Case 2: wind speed variation}

This case refers to the influence of variation in wind speed on the system under the condition of constant load which is $10.20+$ j3.1 MVA. Fig. 8 and Fig. 9 show the curves of system responses under two different conditions of wind speed. In the figures, $\omega, P_{\mathrm{w}}, \omega_{\mathrm{w}}$, and $\theta$ denote the angular frequency of the system, the output of the D-PMSG, the angular velocity and the blade pitch angle of the wind turbine respectively. The black, red and blue curves respectively represent three different situations, in which the ADRVIC and PDVIC are introduced and the VIC is not.

For the high-medium wind speed, which averages 12 $\mathrm{m} / \mathrm{s}$, the curve of wind speed is shown in Fig. 8(a). The inertia responses of the system in different control modes are shown in Fig. 8(b) to Fig. 8(e). Fig. 8(b) shows that the high penetration of wind power in the system results in a relatively large frequency deviation caused by the changing wind speed under the traditional MPPT control, which will seriously influence the frequency stability of the system. The introduction of ADRVIC can reduce the fluctuation range of the system frequency and improve the stability of

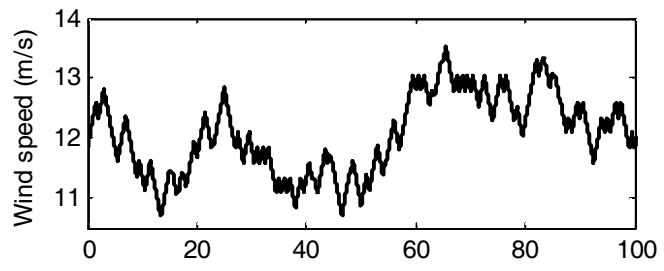

(a) time (s)
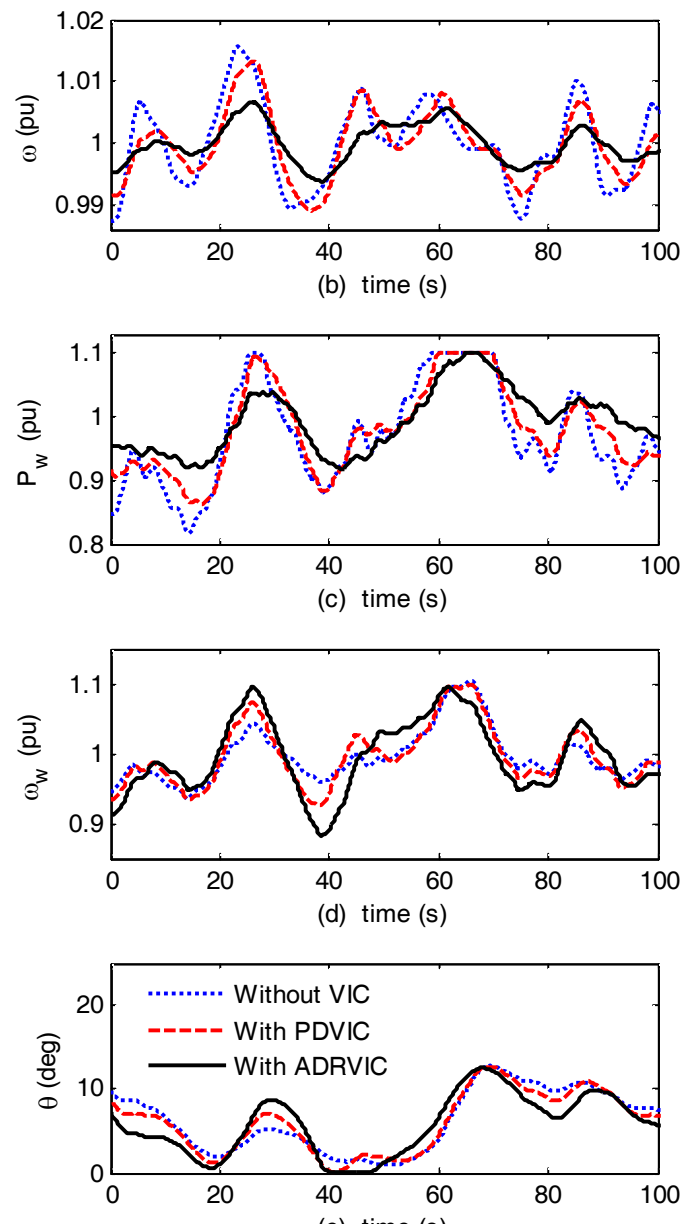

(e) time (s)

Fig. 8. System inertia response for high-medium wind speed 
the system. Furthermore, ADRVIC is more effective than PDVIC. Fig. 8(c) and Fig. 8(d) show that ADRVIC can reduce the impact on the system by smoothing the output of the units; however, this will results in relatively great variation in the rotating speed. When the rotating speed exceeds the rated speed, the pitch angle control will start work to stop it from further rising. The changing curve of the pitch angle is shown in Fig. 8(e).

For the low-medium wind speed, which averages $10 \mathrm{~m} / \mathrm{s}$, the curve of wind speed is shown in Fig. 9(a). The inertia responses of the system in different control modes are shown in Fig. 9(b) to Fig. 9(e). It can be seen from the figures that ADRVIC still functions well under the condition of low-medium wind speed and even has a better

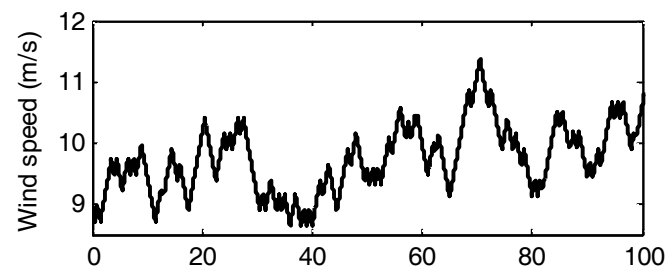

(a) time (s)

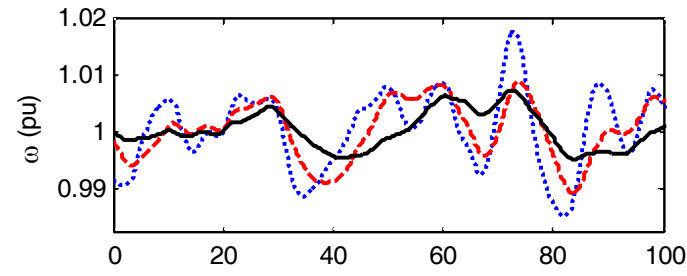

(b) time (s)

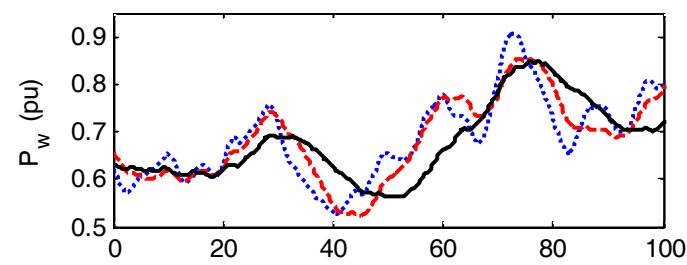

(c) time (s)

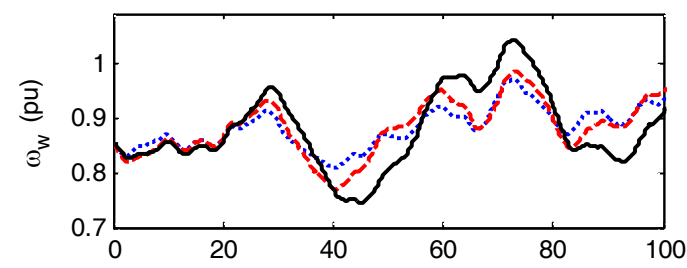

(d) time (s)

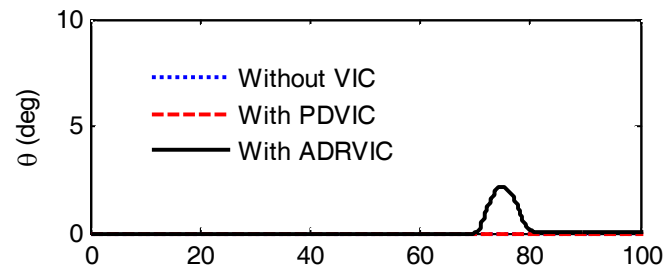

(e) time (s)

Fig. 9. System inertia response for low-medium wind speed
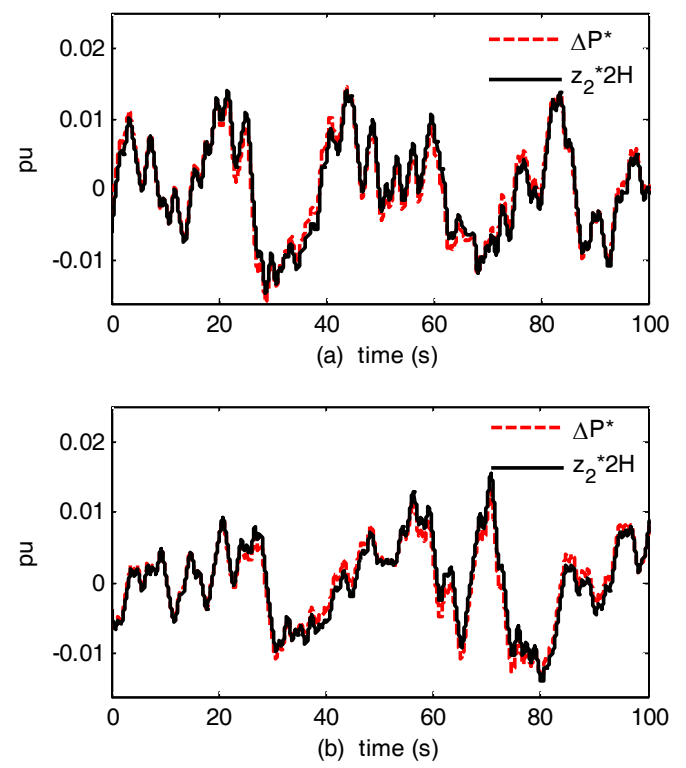

Fig. 10. Observed effect of ESO in ADRVIC under the conditions of high-medium wind speed and lowmedium wind speed

effect than PDVIC.

Fig. 10(a) and Fig. 10(b) show the contrast effect of observation of ESO in ADRVIC under the conditions of high-medium and low-medium wind speed. The red curve denotes the practical power disturbance and the black curve denotes the observed effect of ESO. As shown in Fig. 10, the ESO can still track and estimate the system disturbance accurately under the condition of wind speed disturbance.

\subsection{Case 3: variation in system parameters}

This case refers to the adaptability of ADRVIC to the variation in system parameters. Case 1 and Case 2 demonstrate that ADRVIC has strong ability to suppress the system load disturbance and wind speed disturbance. Parameter $b_{0}$ is taken as actual value $b$ in the algorithm, $b=1 / 2 H=1 / 12$. But in the practical operation of the system, some units quit operation owing to an overhaul while others are connected to the grid due to an increase in load. As a result, the inertia time constant of the system which has been changing is difficult to obtain. For this reason, it is necessary to explore the influence of $b_{0}$ on the control performance of the algorithm.

The system simulation condition is the same as that in Fig. 8, namely the high-medium wind speed which averages $12 \mathrm{~m} / \mathrm{s}$. In this case, the inertia time constant of the system is $6 \mathrm{~s}$; that's $b=1 / 12$, and $b_{0}$ takes the value of $b, 2 b$ and $0.5 b$ respectively. The control performance of ADRVIC is shown in Fig. 11. Fig. 11(a) and Fig. 11(b) show the observed effect of ESO and the frequency response of the system respectively.

It can be seen from the Fig. 11 that the response curves 

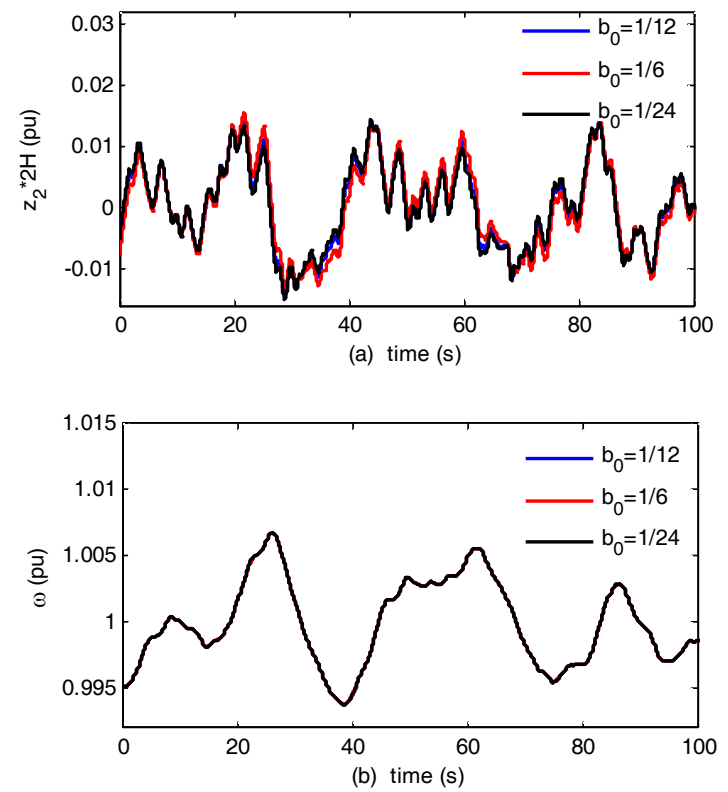

Fig. 11. Response characteristics of ADRVIC with parameter $b_{0}$ varying

of the system overlap completely in the case of different parameters. Therefore, certain variation in parameter b0 will not influence the correct estimation of power disturbance by ESO or the effect of controlling the system frequency by ADRVIC. This illustrates that it is unnecessary to obtain the accurate parameters of the system in ADRVIC, which leads to reducing the dependence of ADRVIC on the grid parameters and ensuring the possibility of its practical application.

\section{Experiments}

\subsection{Test bench}

To demonstrate the possibilities of applying the control algorithm proposed in this paper to the practical D-PMSG, a simulated wind power integrated experiment system is established on the basis of the governor 6RA7025 of Siemens and digital signal processor (DSP) TMS320F2812 of TI Corporation. The hardware structure is shown in Fig. 12.

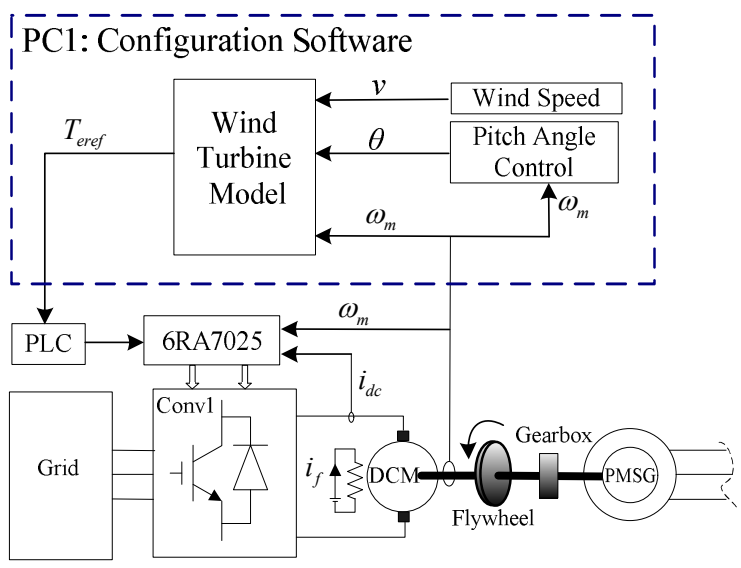

Fig. 13. Control scheme for WTE

The experiment system consists of wind turbine emulator (WTE), PMSG, FPC, interconnection transformer and independent grid. The WTE consists of governor 6RA7025, DC motor, flywheel and gearbox, among which the 6RA7025 controls the output mechanical torque of the DC motor and simulates the output characteristics of the wind turbine. The inertia time constant of the actual MW-level wind turbine generators can be up to $6 \mathrm{~s}$, while the inertia of the actual DC motor is very small (less than $0.5 \mathrm{~s}$ ), so the flywheel is used to simulate the inertia characteristics of the MW-level wind turbine generators. The gearbox is used for connecting the high-speed motor with the lowspeed PMSG.

The control structure of the WTE is shown in Fig. 13. With the configuration software in $\mathrm{PC} 1$, the reference torque of the DC motor is obtained from the samples of motor rotating speed $\omega_{m}$, given wind speed $v$, and practical value of pitch angle $\theta$ according to the mathematical model of wind turbines. And the reference torque command is sent to 6RA7025 through PLC, to perform a closed-loop control of the torque. The FPC implements the control of the generator-side and grid-side converter through TMS320F2812. The control strategy of FPC can be seen from Fig. 1.

The independent power grid is made up of a simulative synchronous generator and equivalent load. The simulative synchronous generator is implemented with an inverter based on the virtual synchronous generator theory [25].

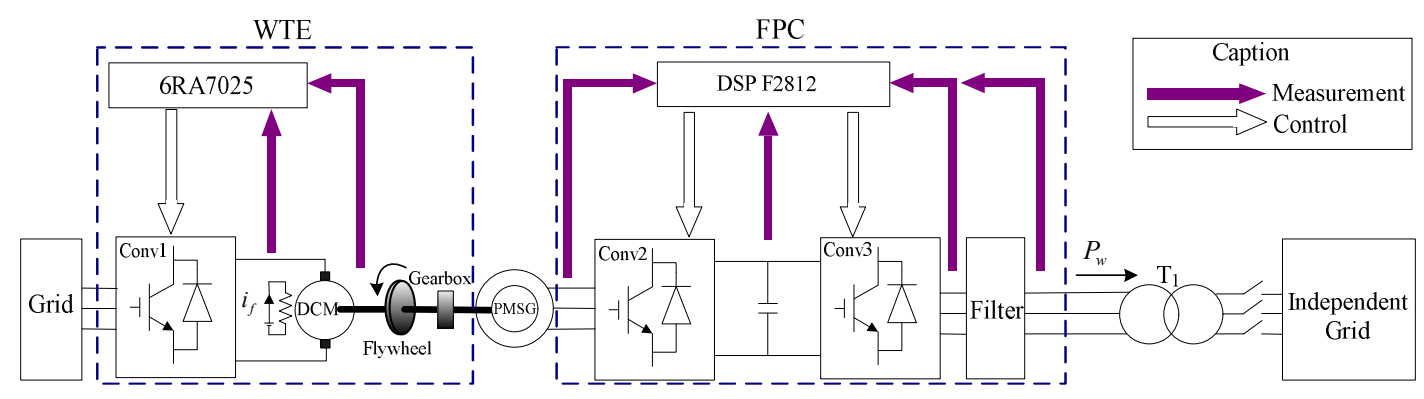

Fig. 12. Experimental platform configuration 


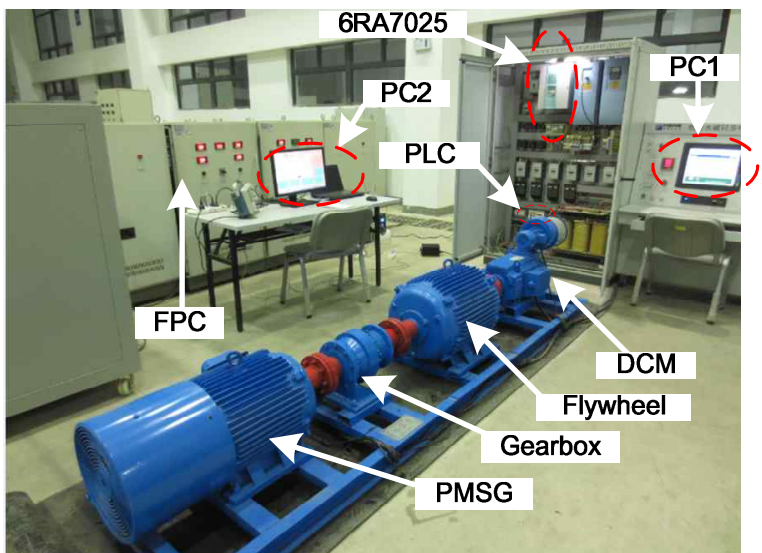

Fig. 14. Test bench

In actual implementation, the armature resistance and synchronous reactance of the synchronous generator are equivalently replaced by the filter reactance, and the inertia characteristics, frequency response characteristics and voltage regulation characteristics of synchronous generators are effectively simulated through the control system.

In the experiment system, the main parameters of the simulated wind turbine generator are: rated power $P_{w n}=6.5$ $\mathrm{kW}$, rated rotating speed $\omega_{w n}=18 \mathrm{rad} / \mathrm{s}$, radius of the wind wheel $R=3.4 \mathrm{~m}$, rated wind speed $v_{n}=9.5 \mathrm{~m} / \mathrm{s}$, and inertia time constant $H_{\mathrm{w}}=5.7 \mathrm{~s}$. The capacity of the independent grid is $S_{n}=8 \mathrm{kVA}$, and the inertia time constant is $H=6 \mathrm{~s}$. The photo of the experiment system is shown in Fig. 14.

\subsection{Experimental results}

Under the condition of low wind speed condition, the inertia response properties of the system can be acquired. In the experiments, the wind speed is constant $7.5 \mathrm{~m} / \mathrm{s}$; the initial load of the system is $7 \mathrm{~kW}$; the system has a sudden addition of load disturbance of $2 \mathrm{~kW}$ when $\mathrm{t}=8 \mathrm{~s}$. The inertia response curves of the system operating on different control modes are shown in Fig. 15. Fig. 15(a) Fig. 15(c) show the response curves of the system frequency, rotating speed of PMSG and output of grid-side converter respectively.

As shown in Fig. 15, the output and rotating speed of the PMSG do not respond to the variations in system frequency under the traditional MPPT control. Thus the system con not get frequency support. However, ADRVIC and PDVIC can get a new coupling relationship established between the wind turbines and the system frequency. When the frequency changes, the D-PMSG can adjust its output and release the kinetic energy of the rotor to support the system frequency. Fig. 15(a) indicates that ADRVIC improves the system frequency more than PDVIC because it can fully release the kinetic energy of the rotor to provide more power support for the system. A comparison between Fig. 15 and Fig. 5 illustrates that the response characteristic
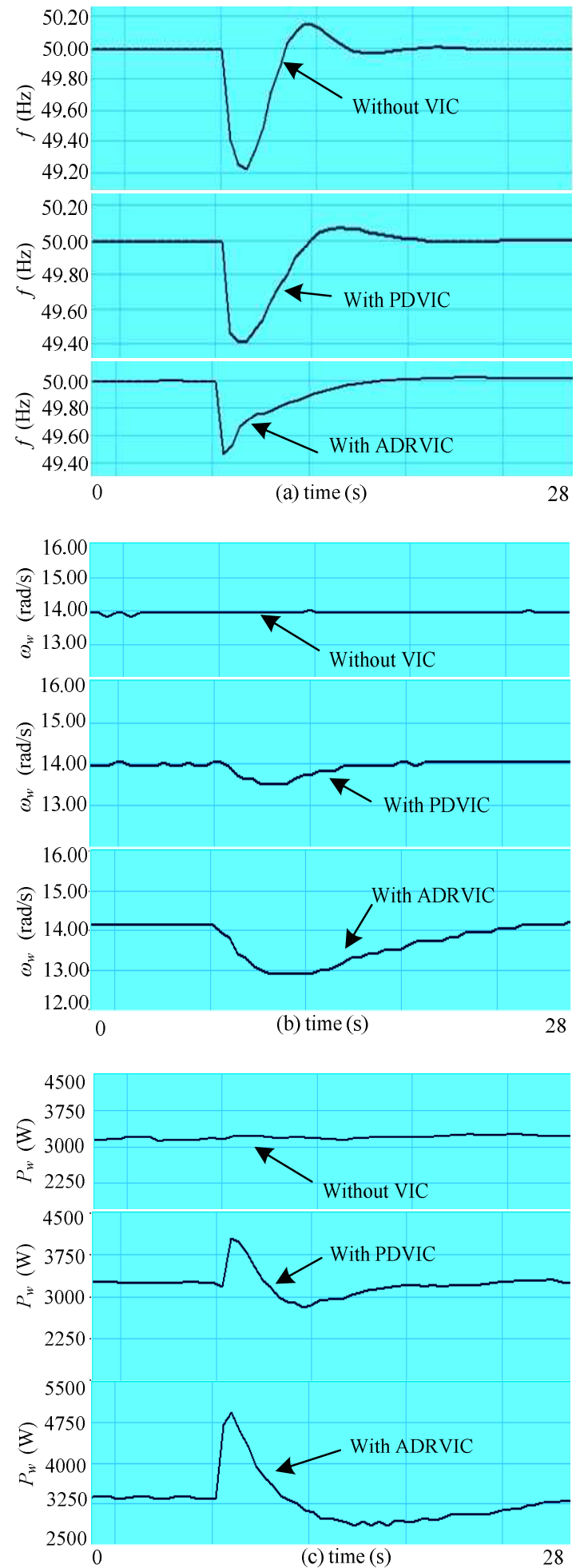

Fig. 15. Inertia response curves of the system in different control modes

curve of the experiment system is almost the same as that of the simulation system in the trend of variation though the two systems are at different power levels. Therefore, the ADRVIC proposed in this paper is effective and feasible in the practical D-PMSG. 


\section{Conclusion}

Based on the design principle of the ADRC, this paper has proposed the ADRVIC method for D-PMSG and compared ADRVIC with conventional PDVIC through simulations and experiments. The results show that both of them can provide inertia and frequency support for the system under the condition of wind speed disturbance or load disturbance.

As ADRVIC participates in the frequency regulation through the observation and compensation of the power disturbance in the system, it can suppress the frequency fluctuation better than PDVIC.

The comparison of the control performances of ADRVIC using different control parameters shows that the phenomenon that the control parameter variation in a certain range has no effect on control of ADRVIC. Therefore, this method is characterized by better adaptability and robustness in practical systems.

\section{Acknowledgements}

This work was supported by the National Basic Research Program of China (973 Program) (No. 2012CB215103), and the National Natural Science Foundation of China (No. 51377167).

\section{References}

[1] M. Chinchilla, S. Arnaltes, and J. C. Burgos, "Control of permanent-magnet generators applied to variablespeed wind-energy systems connected to the grid," IEEE Trans. Power Syst., vol. 21, no. 21, pp. 130-135, Mar. 2006.

[2] J. Morren, J. Pierik, and S. W. Haan, "Inertial response of variable speed wind turbines," Electric Power Systems Research, vol. 76, no.2, pp. 980-987, 2006.

[3] L. Holdsworth, J. Ekanayake, N. Jenkins, "Power system frequency response from fixed speed and doubly fed induction generator based wind turbines," Wind Energy, vol. 7, pp. 21-35, 2004.

[4] H. Lee, J. Kim, D. Hur, and Y. C. Kang, "Inertial Control of a DFIG-based Wind Power Plant using the Maximum Rate of Change of Frequency and the Frequency Deviation," J. Electr. Eng. Technol., vol. 10, no. 2, pp. 496-503, Mar., 2015.

[5] R. G. de Almeida and J. Lopes, "Participation of doubly fed induction wind generators in system frequency regulation," IEEE Trans. Power Syst., vol. 22, no. 3, pp. 944-950, Aug., 2007.

[6] B. G. Rawn, P. W. Lehn, and M. Maggiore, "Control Methodology to Mitigate the Grid Impact of Wind Turbines," IEEE Trans. Energy Convers., vol. 22, no. 2, pp. 431-438, June, 2007.
[7] K. V. Vidyanandan, and N. Senroy, "Primary Frequency Regulation by Deloaded Wind Turbines Using Variable Droop," IEEE Trans. Power Syst., vol. 28, no. 2, pp. 837-846, May., 2013.

[8] J. Morren, S. W. de Haan, W.L. Kling, and J. A. Ferreira, "Wind Turbines Emulating Inertia and Supporting Primary Frequency Control," IEEE Trans. Power Syst., vol. 21, no.1, pp. 433-434, Jan., 2006.

[9] J. M. Mauricio, A. Marano, A. G. Expósito, and J. L. M. Ramos, "Frequency Regulation Contribution Through Variable-Speed Wind Energy Conversion Systems," IEEE Trans. Power Syst., vol. 24, no. 1, pp. 173-180, Feb., 2009.

[10] J. F. Conroy, and R. Watson, "Frequency Response Capability of Full Converter Wind Turbine Generators in Comparison to Conventional Generation," IEEE Trans. Power Syst., vol. 23, no.2, pp. 649-656, May, 2008.

[11] P. Keung, P. Li, H. Banakar, and Boon Teck Ooi, "Kinetic Energy of Wind-Turbine Generators for System Frequency Support," IEEE Trans. Power Syst., vol. 24, no. 1, pp. 279-287, Feb., 2009.

[12] T. H. Nguyen, D.-C. Lee, and J. H. Kang, "Power smoothening control of wind farms based on inertial effect of wind turbine systems," J. Electr. Eng. Technol., vol. 9, no. 3, pp. 1096-1103, May 2014.

[13] M. E. Mokadem, V. Courtecuisse, C. Saudemont, B. Robyns, and J. Deuse, "Fuzzy Logic Supervisor-Based Primary Frequency Control Experiments of a VariableSpeed Wind Generator," IEEE Trans. Power Syst., vol. 24, no. 1, pp. 407-417, May, 2009.

[14] J. Han, "From PID to active disturbance rejection control," IEEE Trans. Ind. Electron., vol. 56, no. 3, pp. 900-906, Mar. 2009.

[15] Z. Gao, "Active Disturbance Rejection Control: A Paradigm Shift in Feedback Control System Design," in publication of the 2006 American Control Conference, Minneapolis, USA, June, 2006.

[16] X. Li and S. Li, "Speed control for a PMSM servo system using model reference adaptive control and an extended state observer," Journal of Power Electronics, vol. 14, no. 3, pp. 549-563, May, 2014.

[17] P. Kundur, Power System Stability and Control, New York: McGraw-Hill, 1994.

[18] J.-W. Jung, V. Q. Leu, D. Q. Dang, H.-H. Choi, and T.-H. Kim, "Sliding mode control of SPMSM drivers: an online gain tuning approach with unknown system parameters," Journal of Power Electronics, vol. 14, no. 5, pp. 980-988, Sept., 2014.

[19] Q. Shi, G. Wang, L. FU, L. Yuan, and H. Huang, "State-space averaging model of wind turbine with PMSG and its virtual inertia control," in Proceedings of the IECON2013, Nov., 1880-1886, 2013.

[20] N. R. Ullah, T. Thiringer, and D. Karlsson, "Temporary Primary Frequency Control Support by Variable Speed Wind Turbines Potential and Applications," 
IEEE Trans. Power Syst., vol. 23, no. 2, pp. 601-612, May. 2008.

[21] H Geng, D Xu, "Stability analysis and improvements for variable-speed multipole permanent magnet synchronous generator-based wind energy conversion system," IEEE Trans. Sus. Energy, vol. 2, no. 4, pp. 459-467, Oct., 2011.

[22] Q. Zheng, L. Q. Gao, and Z. Gao, “On Stability Analysis of Active Disturbance Rejection Control for Nonlinear Time-Varying Plants with Unknown Dynamics," in Proceedings of the of $46^{\text {th }}$ IEEE Conference on Decision and Control, New Orleans, USA, pp. 3501-3506, Dec., 2007.

[23] J. Han, Active Disturbance Rejection Control Technique, Beijing: National Defense Industry Press, 2008.

[24] Z. Gao, "Scaling and Bandwidth-Parameterization Based Controller Tuning," in Proceedings of the 2003 American Control Conference, Denver, USA, pp. 4989-4996, June, 2003.

[25] Q. Zhong, and G. Weiss, "Synchronverters: inverters that mimic synchronous generators," IEEE Trans. Ind. Electron., vol. 58, no. 4, pp. 1259-1267, Apr., 2011.

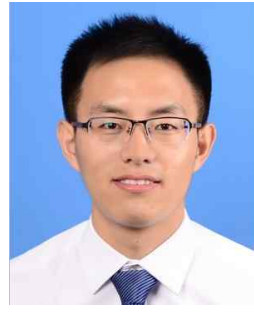

Qiaoming Shi received his B.S. degree in electrical engineering from Xi'an Jiaotong University, China in 2010 . He is currently studying for his Ph.D. degree at Xi' an Jiaotong University. His research interests include the development of wind energy grid integration techniques.

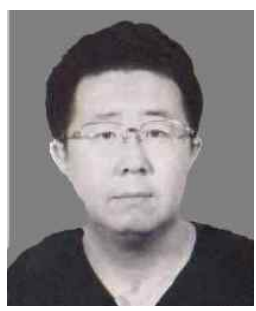

Gang Wang received his B.S. and Ph.D. degrees from Tsinghua University, China in 1993 and 2008 respectively. $\mathrm{He}$ is currently employed as Professor at Naval University of Engineering, China. His research field includes the development of stability control methods for wind power integrated system.

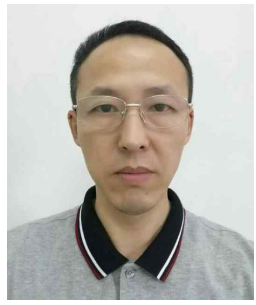

Lijun Fu received the B.S. degree in electrical engineering from Hunan University of Science and Technology in 1989, and the M.S. and Ph.D. degrees from Wuhan University, China respecttively in 1995 and 1997. He is currently employed as Professor at Naval University of Engineering, China. His research field includes the development of stability control methods for Micro-grids.

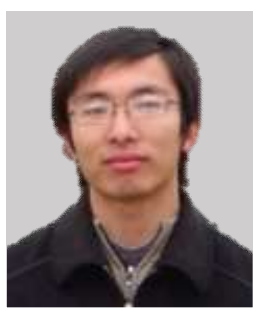

Yang Liu received the B.S. degree in electrical engineering from Wuhan University of Technology in 2008, and the M.S. degree from Shanghai Maritime University, China in 2010. He is currently studying for his $\mathrm{Ph} . \mathrm{D}$. degree at Naval University of Engineering. His research interests include the development of voltage control methods for wind power plants.

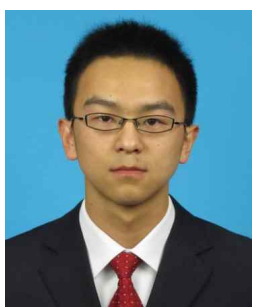

You Wu received his B.S. degree from Tsinghua University, China in 2013. He is currently pursuing his M.S. degree at Naval University of Engineering. His research interests include the development of frequency control methods for wind power integrated system.

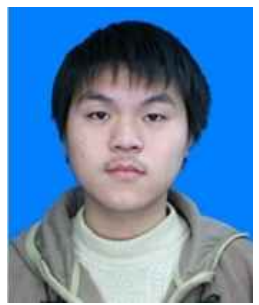

Li Xu received his B.S. degree from Hubei University of Technology, China in 2013 . He is currently pursuing his M.S. degree at Naval University of Engineering. His research interests include the development of voltage control methods for wind power plants. 